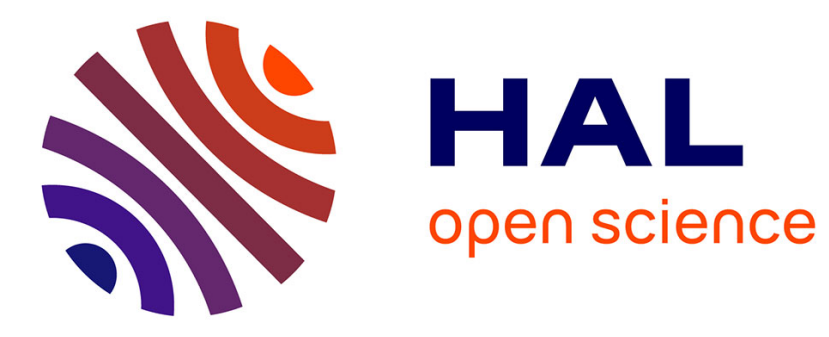

\title{
Mobile TV directed Resource Allocation Scheme for LTE Networks
}

\author{
Ahmad Shokair, Matthieu Crussière, Jean-François Hélard, Youssef Nasser, \\ Oussama Bazzi
}

\section{- To cite this version:}

Ahmad Shokair, Matthieu Crussière, Jean-François Hélard, Youssef Nasser, Oussama Bazzi. Mobile TV directed Resource Allocation Scheme for LTE Networks. 2017 20th International Symposium on Wireless Personal Multimedia Communications (WPMC), Dec 2017, Yogyakarta, Indonesia. hal01756841

\section{HAL Id: hal-01756841 \\ https://hal.science/hal-01756841}

Submitted on 3 Apr 2018

HAL is a multi-disciplinary open access archive for the deposit and dissemination of scientific research documents, whether they are published or not. The documents may come from teaching and research institutions in France or abroad, or from public or private research centers.
L'archive ouverte pluridisciplinaire HAL, est destinée au dépôt et à la diffusion de documents scientifiques de niveau recherche, publiés ou non, émanant des établissements d'enseignement et de recherche français ou étrangers, des laboratoires publics ou privés. 


\title{
Mobile TV directed Resource Allocation Scheme for LTE Networks
}

\author{
Ahmad Shokair ${ }^{* \ddagger}$, Matthieu Crussiere*, Jean-Francois Helard*, Youssef Nasser ${ }^{\dagger}$ and Oussama Bazzi ${ }^{\ddagger}$ \\ * IETR / INSA de Rennes, 20 Ave. des Buttes de Coësmes, Rennes, France \\ \{ashokair, mcrussie, jfhelard\}@insa-rennes.fr \\ $\dagger$ American University of Beirut, Beirut, Lebanon 11072020 \\ yn10@aub.edu.lb \\ $\ddagger$ Lebanese University, Faculty of Sciences, Rafic Hariri University Campus, Hadath, Lebanon \\ obazzi@ul.edu.lb
}

\begin{abstract}
Resource allocation in LTE networks has attracted extensive research efforts. However, linear services (such as Digital TV programs) have been barely considered from the resource allocation perspectives. Indeed, when a linear service is offered to the end-users, the network should satisfy the maximum number of users served rather than maximizing the sum rate capacity. In this paper, linear services oriented resource allocation strategy is proposed. The proposed approach aims at maximizing the users' success rate, based on a minimum required quality of service (QoS) measured in terms of minimum throughput. The proposed method was tested in different scenarios and has shown great robustness against user density, user requirements and reuse factor.
\end{abstract}

\section{INTRODUCTION}

In recent years, mobile users connected to broadband networks have been more and more watching linear services on their mobile phones and tablets. Linear services could be defined as the video services where the end-user watches a program controlled completely by the transmitter. Consequently, broadband networks offering these services have to manage an enormous amount of data traffic. Moreover, these networks have to manage the signal to interference and noise ratio (SINR) due to the inter-cell interference as well as the huge amount of data of such services.

In order to solve the inter-cellular interference problem, different frequency reuse strategies have been proposed in broadband networks. In literature, three main types of those strategies exist: Conventional Frequency reuse, strict Fractional Frequency Reuse (FFR) [1] and Soft Fractional Frequency Reuse (SFR) [2]. The three methods require adequate per resource block (RB) allocation algorithms aiming at satisfying some constraints such as sum rate capacity, minimum capacity, fairness, etc. Among others, the Round Robin (RR) algorithm is presented as the fairness approach. In RR, the Base Station (BS) iteratively allocates the RBs to the different users until all resource blocks are reserved, or the user's constraints are satisfied [3]. This algorithm assures that all users are assigned with RBs, but doesn't consider the channel conditions for each user, so it fails to achieve high rates. Contrarily, Maximum Throughput (MT) starts by allocating the first RB to the user with the best channel conditions, then moves to the second one, and repeats the allocation until all RBs are allocated, or the users' constraints are satisfied. This method assures maximum achievable rate, but at the expense of fairness, since a user with good channel conditions could monopolize most of the RBs, leaving other users with limited or no RBs. However, this method could be used as a comparison level for system capacity. Proportional Fair (PF) [4] is a compromise between the two above methods. It allocates resources according to a formula that considers channel conditions, taking into consideration the number of resource blocks already assigned to that user. Extensive research has been done on the subject for unicast transmission of non-linear services. The reader may refer to the works in [5-8]. However, none of these resource allocation algorithms has dealt with linear services maximizing the number of served users, to the best of the authors' knowledge. In the framework of this work, we tackle the problem of resource allocation for linear services when these have to be offered by LTE network. Here, two possible cases are possible: (1) Multimedia Broadcast Multicast Service (MBMS) mode and (2) the cellular data mode. While the first mode is preferable in practice due to its broadcast nature, it is still not deployed nor implemented in practice. Hence, the second mode presents a suitable solution at the detriment of additional (hungry) resource allocation. However, in linear services, a user needs a minimum capacity to receive the service correctly, and any additional resources allocated to this user will not be reflected in QoS enhancement. Therefore, maximizing the system capacity would be a secondary objective when compared to maximizing the number of users receiving the service.

In this work, we present a new Linear Service Oriented Resource Allocation Strategy (LSORAS), that aims to maximize the number of users receiving the linear service.The proposed algorithm maintains a minimum required capacity for each user, with decent fairness level. The proposed algorithm is compared to RR, PF and MT methods, in terms of average system capacity, average success rate and average fairness index. It has been shown that the proposed algorithm allocates only a portion of the available resources granting a power reduction by idling unused RBs. Moreover, the effect of different frequency reuse patterns has been analyzed in various transmission scenarios including users density. Again, it has been shown that the proposed LSORAS algorithm offers a 
decent performance compared to conventional approaches.

The rest of this paper is organized as follows: In section II, the used model, the assumptions, and the performance metrics are introduced. Section III presents the problem and the new proposed algorithm. In section IV the simulation results are shown and section $\mathrm{V}$ concludes this work.

\section{MODEL DESCRIPTION}

In this section, we describe the system model, the assumptions, and the performance metrics. In our work, we consider the transmission of linear services using Orthogonal Frequency Division Multiplexing (OFDM) technology to $M$ users uniformly distributed in the service area. The BroadBand (BB) network consists of $N_{B S}$ BS sites.

\section{A. Requirements and Assumptions}

It is assumed that the linear service is always available to any user. For a user $m$ to receive the proposed service, a minimum capacity $C^{r e q}$ is required, i.e. $C_{m}^{u s e r} \geq C^{r e q}$. In the broadband network, the linear service is delivered to each connected user separately. The user is attached to the nearest BS, and the transmission power is assumed to be similar for every cell. It is also assumed that the BS has full information about the link quality with each user within the cell boundaries, and can estimate the capacity of the link for each RB.

The BSs are uniformly distributed over the service area, according to a Poisson Point Process (PPP) with the parameter $\lambda$. Voronoi tessellation is used to manage the cell borders. Users positions are also modeled with another PPP with different density. Fig. 1 shows a diagram representing the service area, including base stations and users. The red circle represents the edge of the service area, a cyan line represents an unserved connection and a black line a successful served connection.

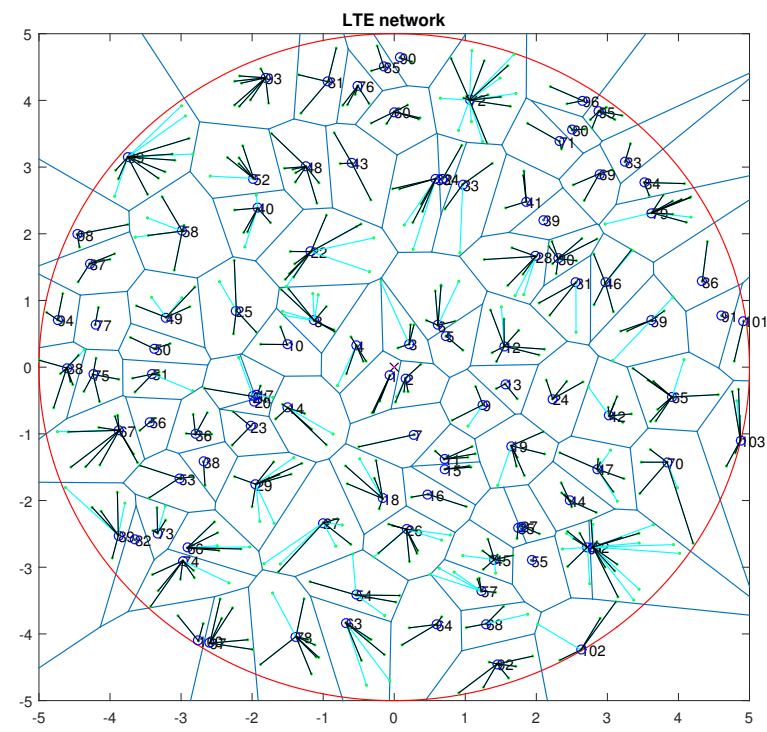

Fig. 1: Modeled broadband network with a PPP describing the position of BSs, users and cells

\section{B. Broadband model}

Each BS transmitter allocates a number of RBs for each connected user. The total number of RBs available at a BS transmitter is denoted by $R B_{\max }$ which depends on the bandwidth of the transmitted signal. The capacity of a single RB $i$ for a user $m$ is given by:

$$
C_{m, i}^{R B}=B_{R B} \log _{2}\left(1+\gamma_{m, i}\right)
$$

where $B_{R B}$ is the bandwidth of a single RB, usually equal to $180 \mathrm{KHz}$ in LTE networks, and $\gamma_{m, i}$ is the SINR at user $m$ for $\mathrm{RB} i$ and it is given by:

$$
\gamma_{m, i}=\frac{P_{t} h_{m, i}\left(\frac{4 \pi f r_{m}}{c}\right)^{-\alpha}}{\sigma^{2}+I_{m, i}}
$$

where $P_{t}$ is the transmitted power, $h_{m, i}$ is a random variable representing the channel for user $m$ and $\mathrm{RB} i$, with exponential distribution of mean $1 / \mu$ i.e. $h \sim \exp (\mu)$. $\alpha$ is the path loss exponent, $f$ represents the operating frequency in $\mathrm{Hz}, \mathrm{c}$ is the speed of light and $r_{m}$ represents the distance between the user $m$ and its serving BS. $\sigma^{2}$ represents the noise power at the receiver and $I_{m, i}$ is the interference power at user $m$ for RB $i$ expressed as:

$$
I_{m, i}=\sum_{j \in \Phi / u_{m}} P_{t} h_{m, i, j}\left(\frac{4 \pi f}{c}\right)^{-\alpha} d_{m, j}^{-\alpha}
$$

where $\Phi$ is the set of all BS transmitters, $u_{m}$ represents user $\mathrm{m}, d_{m, j}$ represents the distance between the receiver $m$ and the interfering transmitter $j$ and $h_{m, i, j}$ is a random variable that represents the channel between user $m$ and the $j t h$ transmitter, for RB $i$. Eq. 2 could then be simplified to:

$$
\gamma_{m, i}=\frac{h_{m, i} r^{-\alpha}}{\tilde{\sigma}^{2}+\sum_{j \in \Phi / b_{0}} h_{m, i, j} d_{m, j}^{-\alpha}}
$$

introducing a normalized noise variance $\tilde{\sigma}$ according to path loss

$$
\tilde{\sigma}^{2}=\frac{\sigma^{2}}{P_{t}\left(\frac{4 \pi f}{c}\right)^{-\alpha}}
$$

The capacity of a user $m$ in the broadband network is assumed to be the sum of capacities of the RBs assigned to this user. Hence it is given by:

$$
C_{m}^{u s e r}=\sum_{i=1}^{R B_{\max }} C_{m, i}^{R B} a_{i, m}
$$

where $a_{i, m}$ is a binary variable that is equal to 1 if $\mathrm{RB} i$ is assigned to user $m$, and equal to 0 otherwise.

Then, the capacity of the cell is defined as the summation of the capacities of all users as follows:

$$
C_{n}^{\text {cell }}=\sum_{m \in \mathscr{\mathscr { M }}_{n}^{\text {cell }}} C_{m}^{\text {user }} b_{m}
$$

where $\mathscr{M}_{n}^{\text {cell }}$ is the set of all users in a cell $n$, and $b_{m}$ is a binary variable indicating whether user $m$ is receiving the cellular linear service or not, i.e. whether the transmitter succeeds or fails to assign enough RBs so that $C_{m}^{u s e r}$ exceeds $C^{r e q}$. 
Finally, the total capacity of the broadband system will be then the sum of capacities of each BS cell, which is given by

$$
C^{S}=\sum_{n=1}^{N_{B S}} C_{n}^{\text {cell }}
$$

where $N_{B S}$ is the number of cells in the BB system.

\section{Performance metrics}

In this work, three main metrics will be used to assess the model and the proposed algorithm: the system capacity, the service success rate and the fairness of the algorithm.

1) System capacity $C^{s}$ : it is the system capacity discussed earlier, and is considered as the maximum throughput achievable by the system.

2) Service success rate $\eta^{s}$ : it is the ratio between the number of users that have access to the service and the total number of users in the service area. A BS has limited resources, thus can serve a limited number of users. In the case of overload, it will deny the service from the excess. In a cell, the success rate can then be stated as:

$$
\eta_{n}^{\text {cell }}=\frac{\text { Number of served users }}{\text { Number of users in the cell }}
$$

which can be also expressed as

$$
\eta_{n}^{\text {cell }}=\frac{\sum_{m \in \mathscr{M}_{n}^{\text {cell }}} b_{m}}{M_{n}^{\text {cell }}}
$$

where $M_{n}^{\text {cell }}$ is the number of users in cell $n$ Hence, the success rate of the system is given by:

$$
\eta^{s}=\frac{\sum_{n=1}^{N_{B S}} \sum_{m \in \mathscr{M}_{n}^{\text {cell }}} b_{m}}{M}
$$

3) Fairness of the algorithm: this factor indicates whether the users in the system are being treated equally. In particular, Jain's fairness index [9], which is the most popular index of fairness, will be used. It is given by:

$$
\mathfrak{J}\left(C_{1}^{\text {user }}, C_{2}^{\text {user }}, \ldots, C_{M}^{\text {user }}\right)=\frac{\left(\sum_{i=1}^{M} C_{m}^{\text {user }}\right)^{2}}{M \sum_{i=1}^{M} C_{m}^{u s e r}{ }^{2}}
$$

For this index, a value of 1 will be the optimum result, where all the users are treated equally, and a value of $1 / M$ is the worst case when only one user monopolizes all the resource blocks. In case of $n$ users, where $k$ users have equal capacities and $(n-k)$ users have no capacity, the fairness index will be $k / n$, which indicates that this index is highly affected by the success rate.

\section{PROBLEM FORMULATION AND PROPOSED ALGORITHM}

\section{A. Problem formulation}

As mentioned in the introduction, our goal is to maximize the success rate described by Eq. (10), with a restriction on the minimum allowed capacity for a served user, and allowing a RB to be assigned to one user only at a time slot. This yields the following optimization of resource allocation problem at the broadband transmitter given by:

$$
\begin{array}{ll}
\max _{\mathbf{a}} & {\left[\sum_{n=1}^{N_{B S}} \sum_{m \in \mathscr{M}_{n}^{\text {cell }}} b_{m}\right]} \\
\text { subject to } & \sum_{i} a_{i, m} \leqslant R B_{\max } \\
& C_{m}^{\text {user }}=\sum_{i=1}^{R B_{\max }} C_{m, i}^{R B} a_{i, m} \\
\forall n \sum_{m \in \mathscr{M}_{n}^{\text {cell }}} a_{i, m} \leqslant 1 . \\
b_{m}= \begin{cases}1, & \sum_{i=1}^{R B_{\max }} a_{i, m} C_{i}^{R B}, m \geq C^{r e q} \\
0, & \text { Otherwise. }\end{cases}
\end{array}
$$

where $\mathbf{a}$ is the matrix holding all $a_{i, m}$. Recall that $b_{m}$ is a binary variable which is equal to 1 if user $\mathrm{m}$ is connected to its associated $\mathrm{BS}$, and equal to zero otherwise. Also recall that $a_{i, m}$ denotes a binary variable that is equal to 1 if $\mathrm{RB}$ $i$ is assigned to user $m$ in its associated cell, $N_{B S}$ denotes the number of cells, $C_{m}^{\text {user }}$ is the achieved capacity of user $m$, $C^{r e q}$ denotes the minimum required capacity for a user to be connected, $R B_{\max }$ denotes the maximum number of resource blocks available in a cell, and $\mathscr{M}_{n}^{\text {cell }}$ denotes the set of all users in cell $n$.

In this problem, $b_{m}$ depends on several other variables in the system, such as SINR, and consequently on the distance from base station $r$, the channel and the interference. It also depends on the number of allocated RBs, and on the choice of those RBs, since each RB has different SINR even for the same user and same base station. The fact that the number of $\mathrm{RBs}$ in a cell is limited increases the complexity of the problem. So whenever a RB is allocated to a user, it will not be available for other users, and the RB pool for the next users is reduced. This correlation between users capacities and variable dependencies indicates that the complexity of the above problem makes its analytical solution prohibitive without losing generality. Alternatively, the choice of $a_{i, m}$ and $b_{m}$ could be based on an algorithm that leads to maximizing the above value. Our proposed algorithm for such solution is described next.

\section{B. Proposed algorithm description}

The primary motivation for our work is the nature of the services. In regular services, the more capacity a user could have, the better the QoS will be, which is reflected in the fact that most algorithms try to maximize the overall capacity of the system. However, when the service is linear, a minimum capacity for a user is needed, which will be enough for a proper reception of this service. Hence, the main idea of our proposed algorithm is to fix a minimum for the user capacity and try to maximize the success rate, which will lead by nature to a higher index of fairness.

Since the number of RBs available in a single cell is limited, a good approach to maximize the number of connected users is to assign RBs to a user, just enough for it to attain $C^{r e q}$. In fact, each user has a vector of SINR values related to the RBs utilized by the serving BS. The proposed algorithm is based on 
assigning the RBs to the users having the best conditions, as this will increase the expected number of served users. Indeed, when the BS allocates the RBs to users with worst condition first, such users will be resource hungry and could drain the RBs for serving a fewer number of demanding users. Besides, the choice of RBs allocated for each user to be the ones with best SINR, will decrease the required RBs needed for this user, and consequently increase the possible number of connected users by making more RBs available.

The proposed LSORAS algorithm for each cell is described in Alg. 1.

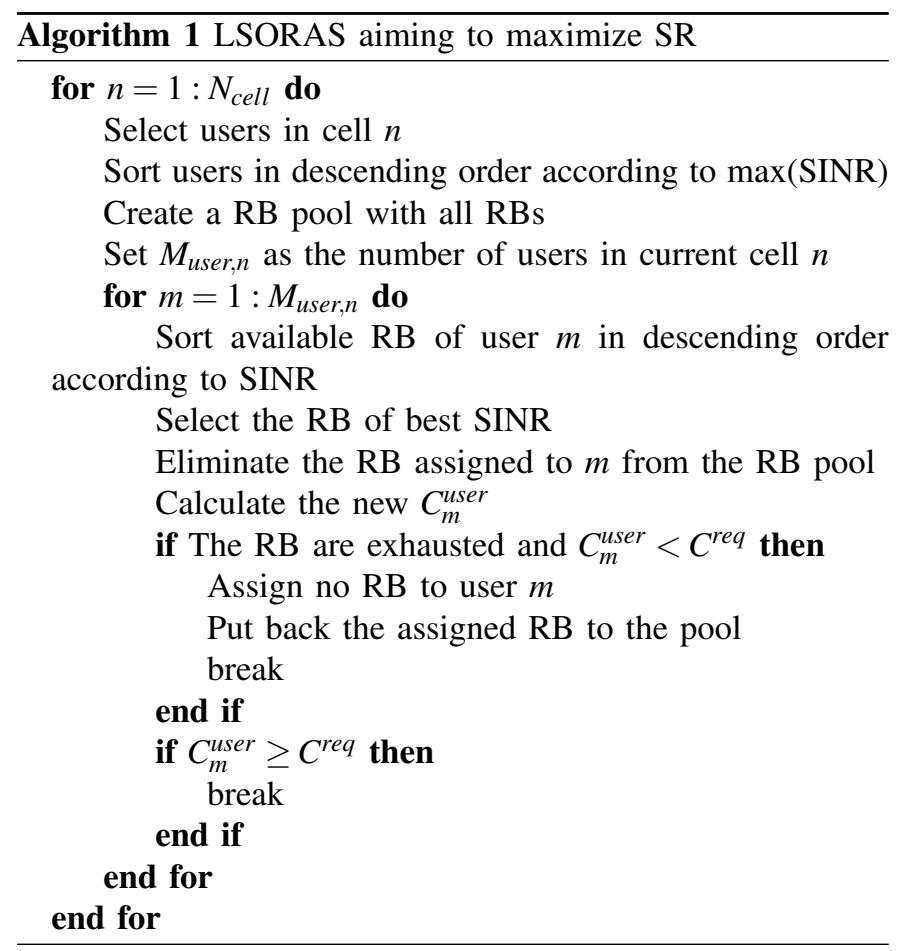

The proposed algorithm satisfies all the points mentioned earlier and consequently leads to the best use of the RBs available in a given distribution. In addition, this algorithm will not assign useless RBs for a user in two cases: (1) when the capacity achieved by a user exceeds $C^{r e q}$, and (2) when allocating the available number of RBs will not satisfy the given user. This condition allows saving RBs to other services within the same cell, or even turn off the unused RBs for power saving.

\section{Simulation RESUlts}

In this section, the proposed algorithm is numerically simulated and compared to MT, RR and PF algorithms to verify its effectiveness. Also, the effect of user density and required capacity on the performance of the algorithm is studied. The impact of frequency reuse is also tested.

\section{A. Simulation setting}

A broadband network model was created, with a PPP of $\lambda=4 / \pi$ representing the position of the BSs, with a Voronoi tessellation for the cell boundaries. The given density is chosen so that the selected service area has on average around 100 BSs. The parameters of the simulations are given in Table I.
TABLE I: Simulation parameters

\begin{tabular}{|l|l|}
\hline \multicolumn{2}{|c|}{ simulation parameters } \\
\hline$\lambda_{B S}$ & $4 / \pi$ \\
EIRP & 1200 Watt \\
$R B_{\max }$ (available RB) & 100 \\
LTE BW & $20 \mathrm{MHz}$ \\
RB BW $B_{R B}$ & $180 \mathrm{kHz}$ \\
Path loss exponent $\alpha$ & 2.8 \\
operating frequency $f$ & $750 \mathrm{MHz}$ \\
Radius of service area & $5 \mathrm{~km}$ \\
\hline
\end{tabular}

\section{B. Probability of coverage}

Figure 2 shows a comparison between the four algorithms in terms of Complementary Cumulative Distribution Function (CCDF), or the probability of coverage, which is the probability of a user to have a capacity that exceeds a particular value. It is clear that the presented resource allocation strategy has a significant advantage over the other algorithms. For a 2 Mbps required capacity, around $80 \%$ of the users succeed to attain this capacity and receive the service correctly, compared to around $50 \%$ with Round Robin and Proportional Fair and around $20 \%$ for Max Throughput. The flat shape of the LSORAS is due to the fixed minimum capacity that a user has to satisfy to be served. That means there will be no users whose capacity is between zero and $C^{r e q}$. It is either zero or some value equal to or (more often) slightly larger than $C^{r e q}$ due to the quantized nature of RB bandwidth, and consequently of the RB capacity.

The figure also shows the difference in probability of coverage between the conventional grid model, with the hexagonal cells, and the more realistic PPP model of the broadband network. In general, the grid model gives an optimistic view on the performance, reflected here in around $10 \%$ increase in success rate compared to the PPP model, which offers a more pessimistic, yet more realistic estimation.

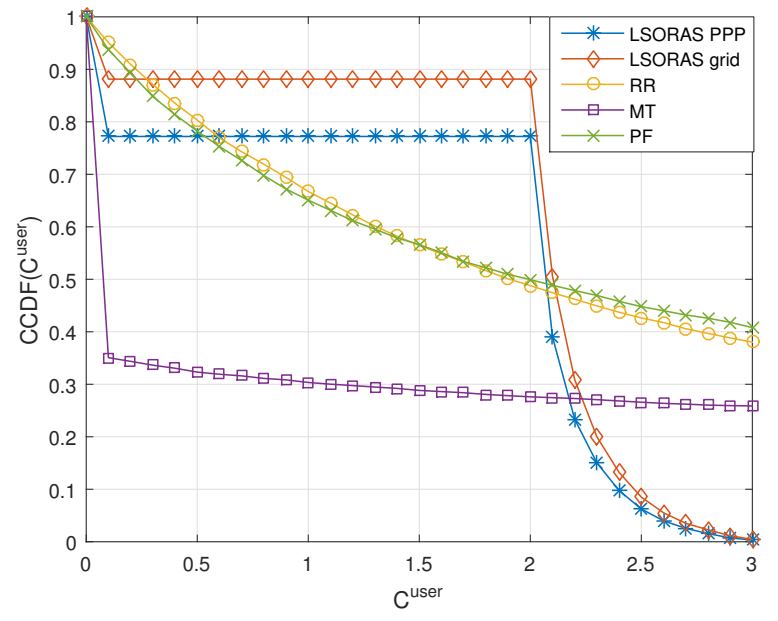

Fig. 2: CCDF of user capacity for 5:1 user to base station ratio, $C^{r e q}=2 \mathrm{Mbps}$ and frequency reuse factor $\Delta=1$

Another significant result is that in contrary to RR, MT, PF and many other algorithms that consume the entire allowed resources, LSORAS manages to reach the success rates using only an average of $43 \%$ of the permitted RBs. This algorithm saves an average of $57 \%$ of the resources, which could be 
assigned to other services, or idled to reduce power consumption.

\section{Effect of user density}

Figures 3, 4 and 5 show the variation of system capacity, success rate and fairness index with respect to the number of users in a service area of 100 base stations respectively. For all the following figures, a PPP is used to distribute the users and base stations. Fig. 3 shows that for a low number of users, LSORAS doesn't achieve high system capacity due to the high dependence on the number of served users, but for a high number of users, LSORAS builds up system capacity and achieves higher values than RR and PF. Fig. 4 shows that the success rate with MT, PF and RR methods dramatically decays with the increase of the number of users. On the other hand, LSORAS maintains higher success rates even with an overload of 50:1 users to BS ratio, while the decay is not as severe as with the other methods. Fig. 5 shows that the LSORAS algorithm maintains a higher degree of fairness, even than Round Robin, since the lower success rate for other algorithms pulls down the fairness index as well, because of the higher number of unserved users.

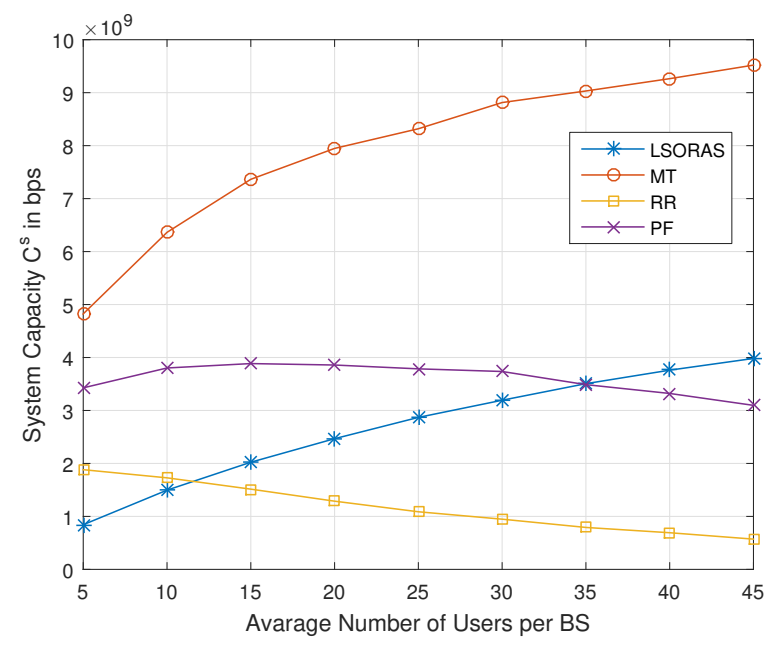

Fig. 3: Average system capacity as a function of number of users for $100 \mathrm{BS}$ in the service area with $\mathrm{C}^{r e q}=2 \mathrm{Mbps}$ and frequency reuse factor $\Delta=1$

\section{Effect of $C^{\text {req }}$}

The quality of the required service is reflected in $C^{r e q}$; the higher the resolution and frame rate, for example, the higher $C^{r e q}$ is set to. The effect of $C^{r e q}$, or the minimum needed capacity for a user to receive the service correctly and on the average success rate is shown in Fig. 6 and 7 respectively.

Fig 6 shows the CCDF of a user capacity, for several values of $C^{r e q}$. It is predictable that, for a low value of $C^{r e q}$, more users will attain the required capacity and vice versa. Nevertheless, Fig 7 shows that LSORAS can maintain a reasonable success rate of around $60 \%$ even with requirements as high as $5 \mathrm{Mbps}$, compared to about $30 \%$ for PF, and about $24 \%$ for both MT and RR algorithms.

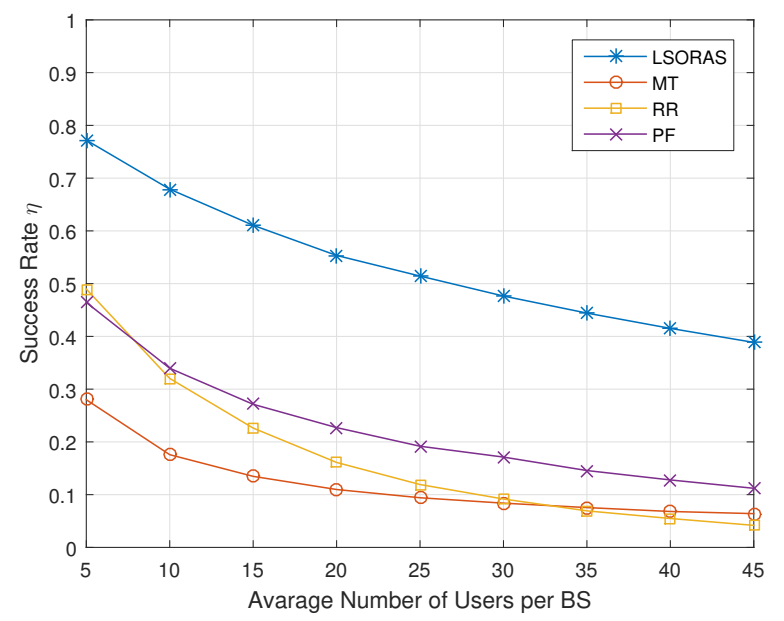

Fig. 4: Average SR vs the number of users for $100 \mathrm{BS}$ in the service area with $C^{r e q}=2 \mathrm{Mbps}$ and reuse factor $\Delta=1$

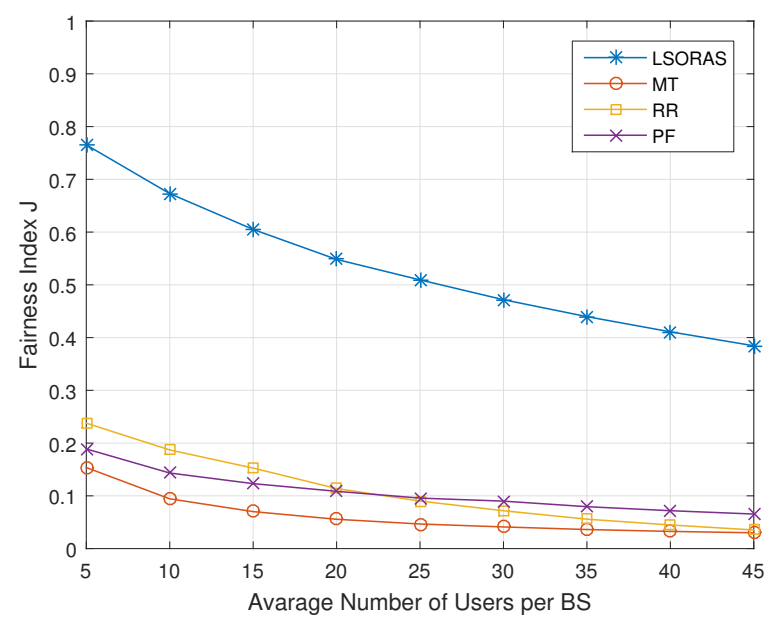

Fig. 5: Average fairness index as a function of number of users for $100 \mathrm{BS}$ in the service area with $\mathrm{C}^{r e q}=2 \mathrm{Mbps}$ and frequency reuse factor $\Delta=1$

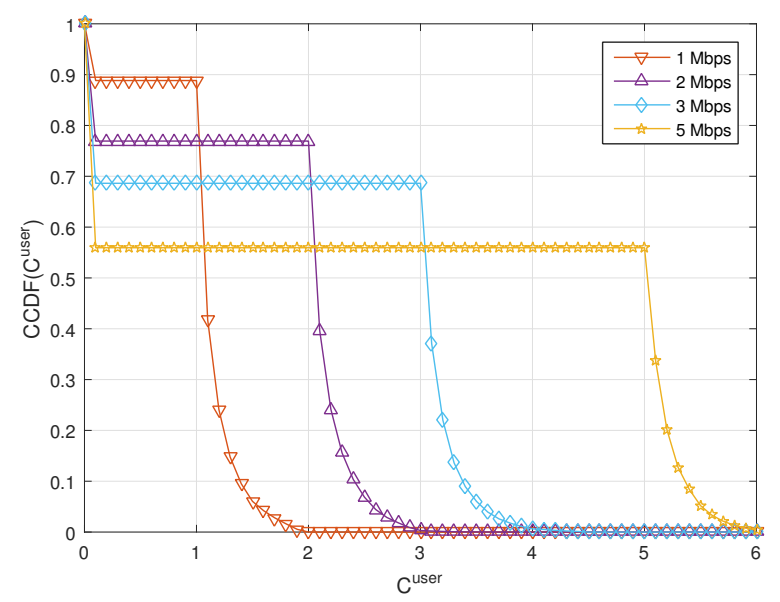

Fig. 6: CCDF of user capacity for 5:1 user to base station ratio, reuse factor $\Delta=1$, and different values of $C^{\text {req }}$

\section{E. The effect of conventional frequency reuse}

The results above were obtained with a system with no frequency reuse, or for a reuse factor of 1 . To study the effect 


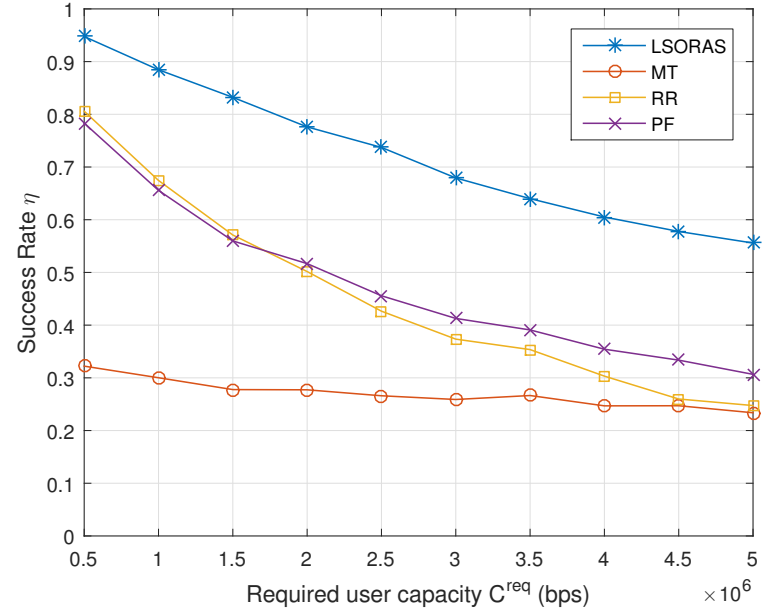

Fig. 7: Average success rate as a function of $C^{r e q}$, with 5:1 user to $\mathrm{BS}$ ratio, and reuse factor $\Delta=1$

of applying frequency reuse method with the LSORAS algorithm on the performance, the conventional frequency reuse method was applied, where each cell selects randomly one of the available bands to operate with. The results for success rate are shown in Figure 8. The idea of the resource allocation strategy depends on the fact that the algorithm has a large pool of RBs to assign to different users. However, when frequency reuse is used, and although the average SINR and capacity of a $\mathrm{RB}$ are boosted due to the reduction of interference presented in (3), the size of the RB pool will shrink, and consequently, the algorithm will lose its maneuverability.

Fig. 8 shows how increasing the reuse factor degrades the success rate in LSORAS, PF, and RR. However, LSORAS keeps a much higher success rate than RR and PF and leads to the conclusion that the reuse factor of 1 is the optimum case. Fairness index, which is not presented here due to paper length limitations, is highly correlated with the success rate, and consequently, will give close results.

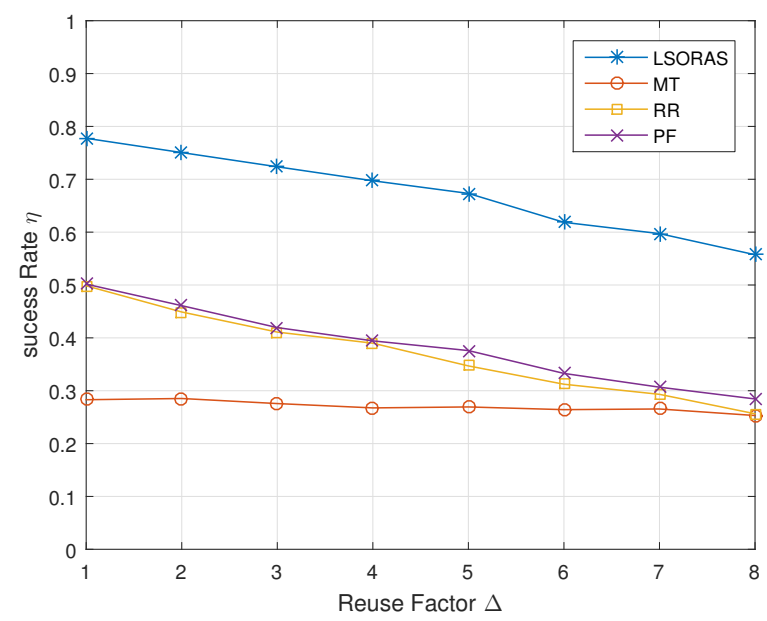

Fig. 8: Average success rate as a function of reuse factor $\Delta$ with $C^{r e q}=2 M b p s$ and 5:1 user to BS ratio

\section{CONCLUSION}

This work has considered the case of linear services provided by a broadband network. We designed LSORAS aiming at maximizing the number of served users in a specific service area. Numerical results have been presented to verify the effectiveness of the proposed algorithm. The results have shown a significant increase in success rate compared to other algorithms. The results also showed the robustness of the algorithm against user density, capacity requirement, and frequency reuse. It also showed that unlike the different algorithms, LSORAS saves a significant portion of RBs for future services.

\section{ACKNOWLEDGEMENT}

This work has received a French state support granted to the Convergence TV project through the $20^{\text {rd }}$ FUI (transverse inter-ministry funding) program. The authors would also like to thank the "Image \& Réseaux" and "Cap Digital" French business clusters for their support of this work.

\section{REFERENCES}

[1] V.Mohite and S.Lahane. "Multi-cell Resource Block Allocation Frequency Reuse Scheme with Optimal Power". In: IJSTE 2.12 (2016), pp. 134-139.

[2] Huawei. "R1-050507: Soft Frequency Reuse Scheme for UTRAN LTE". In: 3GPP TSG RAN WG1 Meeting \#41 (2005).

[3] Z.Tang. Traffic Scheduling for LTE Advanced. 2010.

[4] D.Gesbert. "A Channel Predictive Proportional Fair Schedulaing Algorithm". In: IEEE 6thWorkshop, SPAWC (2005), pp. 620-624.

[5] G.Piro et al. "Two-level downlink scheduling for real-time multimedia services in LTE networks". In: IEEE Transactions on Multimedia 13.5 (2011), pp. 1052-1065.

[6] H.Zhang et al. "Resource Allocation in Spectrum-Sharing OFDMA Femtocells With Heterogeneous Services". In: IEEE Transactions on Communications 62 (2014), pp. 2366-2377.

[7] A.S.Mohamed, M.Abd-Elnaby, and S.A.El-Dolil. "Selforganised dynamic resource allocation scheme using enhanced fractional frequency reuse in long term evolution-advanced relay-based networks". In: IET Communications 10.10 (2016), pp. $1163-1174$.

[8] N.Kumar, A.Ahmad, and D.Prasad. "Survey of downlink control channel resource allocation techniques in LTE". In: 2015 Annual IEEE India Conference (INDICON). 2015, pp. 1-5.

[9] R.Jain, D.M.Chiu, and W.R.Hawe. "A quantitative measure of fairness and discrimination for resource allocation in shared computer system". In: ACM Transaction on Computer Systems cs.NI/9809 (1984), pp. 1-38. 